\title{
Factors influencing the extent of inbreeding depression: an example from Scots pine
}

\author{
PHILIP W. HEDRICK* $\dagger$, OUTI SAVOLAINEN $\ddagger$ \& KATRI KÄRKKÄINEN§ \\ $\dagger$ Department of Biology, Arizona State University, Tempe, Arizona 85287-1501, USA, $\ddagger$ Department of Biology, \\ University of Oulu, PO Box 333, FIN-90571 Oulu, Finland and §Department of Biology and Environmental Science, \\ University of Jyväskylä, PO Box 35, FIN-40351, Jyväskylä, Finland
}

\begin{abstract}
Detailed studies suggest that the level of inbreeding depression may vary between populations. In a study of Scots pine from Finland, the level of inbreeding depression was much lower in northern than in southern populations. We have examined theoretically whether population genetic factors, such as the level of selfing, intensity of selection against heterozygotes or homozygotes, level of mutation, a bottleneck, finite population size, or the level of polyembryony could account for this difference. Higher selfing or stronger selection against heterozygotes in the north, both at biologically reasonable levels, appear to produce changes consistent with the observed differences and we consider these to be the most likely explanations. In addition, the differences could have accumulated by these mechanisms over the age of the northern population, $\approx 100$ generations. Finally, the differences generated by these factors could still be maintained in the face of reasonable levels of gene flow from the south. Such a comprehensive theoretical investigation of this example has given some general insight into the potential influence of these evolutionary factors on the level of inbreeding depression and provides an approach that could be used to understand similar phenomena in other examples.
\end{abstract}

Keywords: bottlenecks, inbreeding depression, lethals, mutation, polyembryony, selfing.

\section{Introduction}

Understanding the factors that influence the level of inbreeding depression has proved to be a challenging and fruitful research arena in recent years, both from an empirical and theoretical perspective (e.g. Charlesworth \& Charlesworth, 1987; Husband \& Schemske, 1996). It appears that most organisms express some level of inbreeding depression for some fitness components, but the estimated inbreeding depression in conifers is among the highest in any group of organisms (e.g. Koski, 1971; Park \& Fowler, 1982; Namkoong \& Bishir, 1987), with estimates indicating that in some species, an average individual may have 10 heterozygous recessive lethals (or lethal equivalents). Examination of the factors that affect inbreeding depression in a group as extreme as conifers may provide insight into inbreeding depression in general.

The frequency of alleles reducing fitness in a population is a function of a number of factors, e.g. the mutation rate, the present and past level of inbreeding, the amount of selection against the alleles as homozygotes and heterozygotes, the present and past effective

*Correspondence. E-mail: philip.hedrick@asu.edu population size, and, in conifers, the level of polyembryony. If these parameters vary among populations, then the expected inbreeding depression (or number of lethal equivalents) may also vary among populations.

Kärkkäinen et al. (1996) showed that populations of Scots pine, Pinus sylvestris, from northern Finland have a statistically significantly lower number of lethals than those from southern Finland. They proposed that this difference in inbreeding depression could be caused by either more self-fertilization in northern than in southern populations, or stronger selection against detrimental alleles in the northern than in the southern populations. However, they did not explore theoretically how large an effect differences in these parameters would potentially have on inbreeding depression. Here we examine these hypotheses, as well as the potential impact of differences in mutation rate, polyembryony, effective population size, and a bottleneck on inbreeding depression. In addition, because the northern populations appear to be only $\approx 100$ generations old (Kärkkäinen et al., 1996), we examine the time to reach equilibrium proportions after colonization from the south.

Before we describe the model, let us introduce the phenomenon of polyembryony. In conifers, multiple embryos may develop in a given ovule but only one 
survives in the mature seed (e.g. Orr-Ewing, 1957; Koski, 1971; Griffin \& Lindgren, 1985; Latta, 1995). In polyembryony as found in conifers, all the embryos within an ovule contain the same maternal gamete, whereas the fertilizing paternal gametes are assumed to be independent of each other when there is outcrossing and reflect segregation from the maternal genotype, i.e. are not necessarily identical, when there is self-fertilization. Although there can be more than two embryos per ovule, we will assume below that there are either one or two embryos per ovule both because these are by far the largest classes in Scots pine and because examination of some theoretical examples (Hedrick, unpub.) demonstrates that this adequately describes the phenomenon.

\section{The model}

\section{Estimate of number of lethal equivalents}

Let us assume that an individual heterozygous at a single locus with one embryo per ovule is selfed. Assume that the fitnesses (used here as synonymous with viability) of genotypes $A_{1} A_{1}, A_{1} A_{2}$ and $A_{2} A_{2}$ are 1 , $1-h s$ and $1-s$, respectively, where $s$ indicates the level of selection against the homozygote $A_{2} A_{2}(s=1$ for a lethal) and $h$ indicates the level of dominance. The average fitness (viability) of the selfed progeny is then

$\bar{w}_{\mathrm{S} .1}=1 \quad \frac{1}{4} s(1+2 h)$.

For $n$ such identical and independent loci, the fitness is

$\bar{w}_{\text {S.1.n }}=\left[\begin{array}{ll}1 & \frac{1}{4} s(1+2 h)\end{array}\right]^{n}$

and the proportion of ovules with no seeds is

$E_{\mathrm{S} .1 . n}=1 \quad \bar{w}_{\mathrm{S} .1 . n}$.

When there are two embryos per ovule, at most only one can survive as a seed. However, at an earlier stage, depending upon their relative fitnesses at locus $A$, either both survive, one or the other of them survives, or both die. When both survive, then we assume that a random embryo matures into a seed. As an example, let us assume that the genotypes of the gametes from the maternal plant are both $A_{1}$ and that the two pollen grains have alleles $A_{1}$ and $A_{2}$. Because the $A_{1} A_{1}$ embryo survival is assumed to be unity, the probability that both embryos die is 0 , the probability that only the $A_{1} A_{1}$ embryo survives is $h s$, the probability that only the $A_{1} A_{2}$ embryo survives is 0 , and the probability the both survive is $1-h s$. If it is assumed that in this last case a random embryo survives, then the proportions of $A_{1} A_{1}$ and $A_{1} A_{2}$ surviving overall are $(1+h s) / 2$ and $(1-h s) / 2$, respectively. The proportions of embryos that survive for this and other combinations of maternal and paternal gametes are given in Table 1.

Therefore, for two embryos per ovule, when a heterozygous individual is selfed, the fitness is

$\bar{w}_{\mathrm{S} .2}=1 \quad \frac{1}{8} s^{2}\left(2 h^{2}+2 h+1\right)$.

For $n$ such loci, the fitness is

$\bar{w}_{\text {S.2.n }}=\left[\begin{array}{ll}1 & \frac{1}{8} s^{2}\left(2 h^{2}+2 h+1\right)\end{array}\right]^{n}$

and the proportion of ovules with no seeds is

$E_{\mathrm{S} .2 . n}=1 \quad \bar{w}_{\mathrm{S} .2 . n}$.

If there is a proportion $x_{1}$ of ovules with one embryo and a proportion $x_{2}$ of ovules with two embryos, then the fitness is

$\bar{w}_{\mathrm{S}}=x_{1} \bar{w}_{\mathrm{S} .1}+x_{2} \bar{w}_{\mathrm{S} .2}$.

If $s=1$, then this expression becomes

$\bar{w}_{\mathrm{S}}=\frac{1}{8}\left[\begin{array}{llll}7 & x_{1} & 2 h\left(x_{1}+h+1\right. & \left.x_{1} h\right)\end{array}\right]$.

Table 1 The proportions of the different genotypes surviving when there are two embryos per ovule

\begin{tabular}{|c|c|c|c|c|}
\hline \multirow[b]{3}{*}{ Paternal gametes } & \multicolumn{4}{|c|}{ Maternal gamete } \\
\hline & \multicolumn{2}{|c|}{$A_{1}$} & \multicolumn{2}{|c|}{$A_{2}$} \\
\hline & Genotype & Frequency & Genotype & Frequency \\
\hline$A_{1}, A_{1}$ & $A_{1} A_{1}$ & 1 & $A_{1} A_{2}$ & $1-h^{2} s^{2}$ \\
\hline$A_{1}, A_{2}$ & $A_{1} A_{1}$ & $(1+h s) / 2$ & $A_{1} A_{2}$ & $(1-h s)(1+s) / 2$ \\
\hline & $A_{1} A_{2}$ & $(1-h s) / 2$ & $A_{2} A_{2}$ & $(1+h s)(1-s) / 2$ \\
\hline$A_{2}, A_{2}$ & $A_{1} A_{2}$ & $1-h^{2} s^{2}$ & $A_{2} A_{2}$ & $1-s^{2}$ \\
\hline
\end{tabular}


The proportion of empty seeds for $n$ loci is

$E_{\mathrm{S} . n}=1 \quad\left(\bar{w}_{\mathrm{S}}\right)^{n}$.

This equation can be solved to obtain the estimate of the number of lethal equivalents as

$n_{\mathrm{le}}=\frac{\ln \left(1 \quad E_{\mathrm{S} . n}\right)}{\ln \bar{w}_{\mathrm{S}}}$.

If it is assumed that there is some estimate of random environmental mortality from the observed proportion of empty seeds from randomly outcrossed, unrelated individuals, $E_{\mathrm{O}}^{*}$, then the overall observed proportion of empty seeds from selfing, $E_{\mathrm{S}}^{*}$, assuming multiplicative effects, is

$$
\left(\begin{array}{ll}
1 & E_{\mathrm{S}}^{*}
\end{array}\right)=\left(\begin{array}{ll}
1 & E_{\mathrm{S} . n}
\end{array}\right)\left(\begin{array}{ll}
1 & E_{\mathrm{O}}^{*}
\end{array}\right)
$$

and

$\left(\begin{array}{ll}1 & E_{\mathrm{S} . n}\end{array}\right)=\frac{\left(\begin{array}{ll}1 & E_{\mathrm{S}}^{*}\end{array}\right)}{\left(\begin{array}{ll}1 & E_{\mathrm{O}}^{*}\end{array}\right)}$

This value can then be used in expression (4b) to estimate the number of lethal equivalents.

The primary selfing rate $(S)$, the rate of selfing at fertilization before any selection, can be estimated from

$S=\frac{S_{\mathrm{m}}}{1 \quad\left(1 \quad S_{\mathrm{m}}\right) \delta}$,

where $S_{\mathrm{m}}$ is the selfing rate measured at the seed stage after some selection could have occurred and $\delta=1 \quad\left(\bar{w}_{\mathrm{S}} / \bar{w}_{\mathrm{O}}\right)$ where $\bar{w}_{\mathrm{O}}$ is the fitness of outcrossed zygotes (see Lande et al., 1994).

\section{Population frequencies}

Let us assume mutation is unidirectional with the mutation rate from $A_{1}$ to $A_{2}$ equal to $u$. Further, let the (primary) rate of selfing in the population be $S$ and the rate of outcrossing be $1-S$. Therefore, selection will act to reduce the frequency of the detrimental allele and mutation will increase its frequency, leading to a mutation-selection equilibrium. The proportion of selfing will influence the equilibrium allele frequency based on the mutation-selection balance (Lande \& Schemske, 1985) as well as the rate of allele frequency change (Hedrick, 1985, p. 158).

If we let the frequencies of alleles $A_{1}$ and $A_{2}$ be $p$ and $q$, then Table 2 gives the expected progeny frequencies when there is only one embryo per ovule. Using these values, assuming that the frequency of parental genotypes $A_{1} A_{1}$, $A_{1} A_{2}$ and $A_{2} A_{2}$ are $P, H$ and $Q$, respectively, and that the fitnesses of these genotypes are as given above, the frequencies of the genotypes in the progeny are

$$
P_{1}^{\prime}=\frac{(1 \quad u)^{2}}{\bar{w}_{1}}\left[S\left(P+\frac{1}{4} H\right)+\left(\begin{array}{ll}
1 & S
\end{array}\right) p^{2}\right],
$$

$$
\begin{aligned}
& H_{1}^{\prime}=\frac{(1 \quad u)(1 \quad h s)}{\bar{w}_{1}}\left\{S\left[2 u P+\frac{1}{2} H(1+u)\right]\right. \\
& \left.+(1 \quad S)\left[P(q+2 u p)+\frac{1}{2} H(1+2 u p)+Q p\right]\right\}
\end{aligned}
$$

and

$$
\begin{aligned}
Q_{1}^{\prime}= & \frac{(1 \quad s)}{\bar{w}_{1}}\left\{S \left[\left(P u^{2}+\frac{1}{4} H\left(1+u^{2}\right)+Q\right]\right.\right. \\
& \left.+(1 \quad S)(q+u p)^{2}\right\}
\end{aligned}
$$

where $\bar{w}_{1}$ is the sum of the right side of the above equations when multiplied by $\bar{w}_{1}$. The frequency of empty seeds, those with no viable embryo, is

$$
E_{1}=1 \quad \bar{w}_{1} .
$$

\begin{tabular}{|c|c|c|c|c|c|}
\hline & \multicolumn{2}{|c|}{ Maternal parent } & \multicolumn{3}{|c|}{ Progeny } \\
\hline & Genotype & Frequency & $A_{1} A_{1}$ & $A_{1} A_{2}$ & $A_{2} A_{2}$ \\
\hline Selfing $(S)$ & $\begin{array}{l}A_{1} A_{1} \\
A_{1} A_{2} \\
A_{2} A_{2}\end{array}$ & $\begin{array}{l}P \\
H \\
Q\end{array}$ & $\begin{array}{c}(1-u)^{2} \\
(1-u)^{2} / 4 \\
0\end{array}$ & $\begin{array}{c}2 u(1-u) \\
\left(1-u^{2}\right) / 2 \\
0\end{array}$ & $\begin{array}{c}u^{2} \\
(1+u)^{2} / 4 \\
1\end{array}$ \\
\hline Outcrossing $(1-S)$ & $\begin{array}{l}A_{1} A_{1} \\
A_{1} A_{2} \\
A_{2} A_{2}\end{array}$ & $\begin{array}{l}P \\
H \\
Q\end{array}$ & $\begin{array}{c}p(1-u)^{2} \\
p(1-u)^{2} / 2 \\
0\end{array}$ & $\begin{array}{c}(1-u)(q+2 u p) \\
(1-u)(1+2 u p) / 2 \\
p(1-u)\end{array}$ & $\begin{array}{c}u(q+u p) \\
(1+u)(q+u p) / 2 \\
q+u p\end{array}$ \\
\hline
\end{tabular}

Using the analogous frequencies of gametes as in Table 1 for two embryos per ovule, then the frequencies of the three genotypes are

Table 2 The frequency of different types of progeny when there is either selfing or outcrossing and one embryo per ovule 


$$
\begin{aligned}
& P_{2}^{\prime}=\frac{(1 \quad u)^{2}}{\bar{w}_{2}}\left\{S \left[\left(P(1+u h s)+\frac{1}{8} H(2+h s+u h s)\right]\right.\right. \\
& \left.+\left(\begin{array}{ll}
1 & S
\end{array}\right) p^{2}[1+q h s+u p h s]\right\}, \\
& H_{2}^{\prime}=\frac{\left(\begin{array}{lll}
1 & u
\end{array}\right)(1 \quad h s)}{\bar{w}_{2}}\{S[P u(2+h s+u s) \\
& \left.+\frac{1}{8} H(1+u)(4+2 h s+s \quad u s)\right] \\
& +(1 \quad S)\left[P \left(p(1 \quad u)(q+u p)+(1+h s)(q+u p)^{2}\right.\right. \\
& \left.+p^{2} u(1 \quad u)(1+h s)+p u(q+u p)(1+s)\right) \\
& +\frac{1}{2} H\left(p(1 \quad u)(q+u p)+(q+u p)^{2}(1+h s)\right. \\
& \left.+p^{2}\left(1 \quad u^{2}\right)(1+h s)+p(1+u)(q+u p)(1+s)\right) \\
& +Q p(1+p h s \quad u p h s+q s+u p s)]\}
\end{aligned}
$$

and

$$
\begin{aligned}
Q_{2}^{\prime}= & \frac{(1 \quad s)}{\bar{w}_{2}}\left\{S \left[\left(P u^{2}\left(\begin{array}{lll}
1 & u h s+h s & u s
\end{array}\right)\right.\right.\right. \\
& +\frac{1}{8} H(1+u)^{2}\left(\begin{array}{ll}
2 & s+u s+h s \quad u h s)+Q(1+s)
\end{array}\right] \\
& +\left(\begin{array}{ll}
1 & S)(q+u p)\left[P u+\frac{1}{2} H(1+u)+Q\right.
\end{array}\right] \\
& \times[1+p h s \quad u p h s+q s+u p s]\}
\end{aligned}
$$

where $\bar{w}_{2}$ again is the sum of the right side of the above equations when multiplied by $\bar{w}_{2}$ and

$E_{2}=1 \quad \bar{w}_{2}$.

If we assume that the number of lethal equivalents $\left(n_{\mathrm{le}}\right)$ in an individual is the number of loci for which they are heterozygous for a lethal allele, then

$n_{\mathrm{le}}=H n^{*}$,

where $n^{*}$ is the number of loci that can carry a lethal allele. The number of lethal equivalents is therefore a direct function of the heterozygosity obtained from the above equations. Only if $s \ll 1$, will a substantial proportion of detrimental alleles be in homozygotes, resulting in an underestimate of the number of lethal equivalents.

Using the number of lethal equivalents estimated from (4b) and the level of equilibrium heterozygosity from expressions (7b) and (8b), then from expression (9a), an estimate of the number of loci with lethals is

$n^{*}=\frac{n_{\mathrm{le}}}{H}$.

\section{Results}

\section{Estimate of level of polyembryony}

Sarvas (1962) estimated the frequencies of $P$. sylvestris ovules with different numbers of embryos from five Finnish locations. For the 840 ovules examined, 392, 394, 52 and 2 had 1, 2, 3 and 4 embryos, respectively, with a mean of 1.60 embryos per ovule. Below we will examine the effect of variable numbers of embryos and then, following Savolainen et al. (1992), assume that there were only ovules with either one or two embryos and that the mean number of embryos was 1.6 , with 0.4 of the ovules having one embryo (equal to $x_{1}$ below) and 0.6 with two embryos $\left(x_{2}\right)$.

\section{Estimate of number of lethal equivalents}

As discussed above, let us assume that the proportion of ovules with one embryo is $0.4\left(x_{1}\right)$ and that the remainder have two embryos $\left(x_{2}=0.6\right)$ (Sarvas, 1962; Savolainen et al., 1992). Further, assume that here we are considering only lethals, so that $s=1$, and that their average dominance is reflected by $h=0.02$, the best general estimate obtained from Drosophila melanogaster (e.g. Simmons \& Crow, 1977; Charlesworth \& Charlesworth, 1987). Then from expression (3b), $\bar{w}_{\mathrm{S}}=0.818$. In the samples from the south of Finland, $E_{\mathrm{O}}^{*}=0.248$ and $E_{\mathrm{S}}^{*}=0.865$ (Kärkkäinen et al., 1996) so that using expressions (4b) and (5), the number of lethal equivalents is estimated to be 8.54 . In the samples from northern Finland, $E_{\mathrm{O}}^{*}=0.237$ and $E_{\mathrm{S}}^{*}=0.754$ (Kärkkäinen et al., 1996) and the estimate of the number of lethal equivalents is 5.63. In other words, the estimated number of lethal equivalents in the north is $65.9 \%$ of that in the south, i.e. there is a $34.1 \%$ reduction in the number of lethal equivalents. These estimates are close to the estimates obtained by Kärkkäinen et al. (1996) using the approach of Koski (1971), and higher than the estimate they obtained using the approach of Sorensen (1969). However, the approach of Koski does not incorporate environmental mortality and the approach of Sorensen does not incorporate polyembryony, making the new estimates more comprehensive.

\section{Estimate of primary selfing rate}

The estimated primary selfing rates from the south and north Finnish populations are 0.016 and 0.071 , respectively (Kärkkäinen et al., 1996). Using expression (6) and assuming that $\bar{w}_{\mathrm{S}}=1 \quad E_{\mathrm{S}}^{*}$ and $\bar{w}_{\mathrm{O}}=1 \quad E_{\mathrm{O}}^{*}$, the estimates of the primary selfing rates in the south and north are 0.083 and 0.192 , respectively. We should note 
that these estimates are potentially influenced by two different factors but in opposite directions. First, expression (6) does not include the effects of polyembryony and therefore may overestimate the rate of primary selfing (Lande et al., 1994). Secondly, we have used observed estimates of empty seeds from open-pollination rather than only outcrossed seeds, which should lead to an underestimate of the primary selfing rate (however, in Scots pine these values are quite close; Kärkkäinen et al., 1996). Because these effects probably counter each other to some extent and we are primarily interested in the relative differences in the south and north populations, we feel these estimates are reasonable for our analysis below.

\section{Effects of various parameters on equilibrium heterozygosity}

The above sets of equations for genotypic frequencies can be iterated until there is an equilibrium to examine the effects of the various parameters. In the following examples, the rate of mutation per generation is assumed to be either $10^{-4}$ or $10^{-5}$, rates consistent with the discussion for plant lethal mutation rates in Lande et al. (1994) and Kärkkäinen et al. (1996). First, Fig. 1 gives the equilibrium heterozygosity frequency, $H_{\mathrm{e}}$, for different levels of two embryos $\left(x_{2}\right)$ and three levels of selfing $S$ when $u=10^{-5}, s=1$, and $h=0$. When $x_{2}=0$ (only one embryo per ovule), and $S>0.05$, then $H_{\mathrm{e}} \approx 4 u / S$ (Lande \& Schemske, 1985). When $x_{2}=1$ (only two embryos per ovule), then $H_{\mathrm{e}} \approx 16 u / 3 S$. When there is a proportion $x_{2}$ of ovules with two embryos, then $H_{\mathrm{e}} \approx 4 u\left(3+x_{2}\right) / 3 S$. Overall, the level of equilibrium heterozygosity when $x_{2}$ increases from 0 to 1 is increased by $41 \%$ when $S=0$, and is increased by $\approx 33.3 \%$ when $S>0.05$. The equilibrium frequency of empty seeds, $E$, when $x_{2}=0$ is equal to $u$. When $x_{2}=1$, then $E \approx 2 u / S$ if $S>0.05$.

The level of dominance has a large effect on the equilibrium heterozygosity (Fig. 2). For example, when $h$ increases only from 0 to 0.02 for $u=10^{-5}$, the equilibrium heterozygosity decreases $34.7 \%$. On the other hand, an increase in selection against homozygotes an order of magnitude larger (not shown), from $s=0.8$ to 1.0 for $u=10^{-5}$, results in a decrease in equilibrium heterozygosity of only $8.9 \%$. The basis of this difference in the effectiveness of selection against heterozygotes and homozygotes becomes clear when it is realized that for most of the situations examined here, approximately one to two orders of magnitude more alleles are in heterozygotes than in homozygotes. The level of selfing also has a large effect and even a small increase in selfing will reduce the equilibrium heterozygosity considerably (Fig. 3). For example, when the level of selfing is

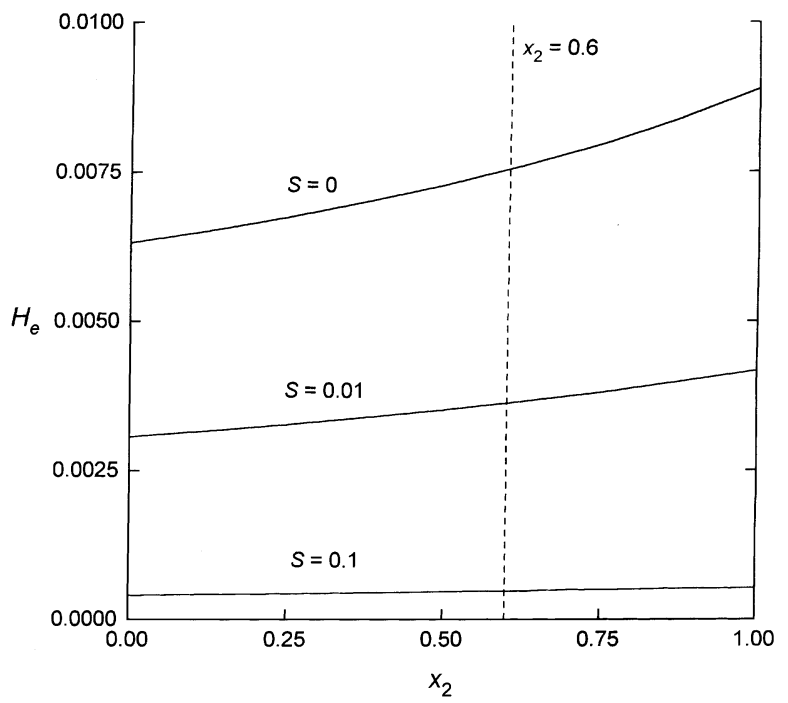

Fig. 1 The equilibrium level of heterozygosity for different levels of two embryos $\left(x_{2}\right)$ and three levels of selfing when there is a completely recessive lethal $(s=1, h=0)$ and a mutation rate of $10^{-5}$.

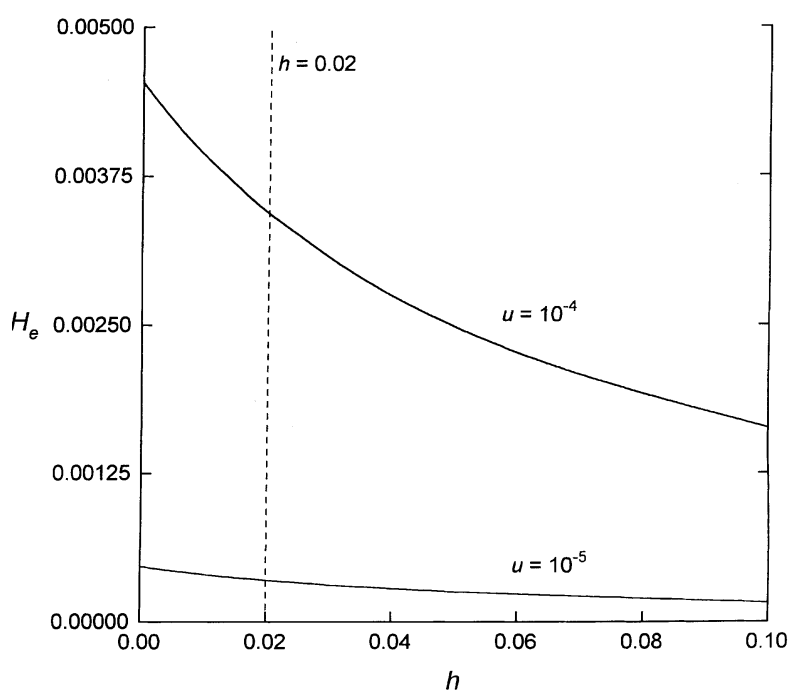

Fig. 2 The equilibrium level of heterozygosity for different levels of dominance and two mutation rates for a lethal $(s=1), x_{2}=0.6$, and a selfing rate of 0.1 .

increased from $S=0$ to $S=0.05$ and $u=10^{-5}$, then the equilibrium heterozygosity is reduced by $87.8 \%$.

\section{Estimate of the number of loci capable of producing recessive lethal mutations}

The number of loci capable of producing recessive lethal mutations, $n^{*}$, can be estimated with expression (9b), which is a function of the estimate of the number of lethal equivalents and the equilibrium heterozygosity. 




Fig. 3 The equilibrium level of heterozygosity for different levels of selfing and two mutation rates for a recessive lethal $(s=1, h=0)$ and $x_{2}=0.6$.

To obtain the general range of these estimates, let us use the estimate of lethal equivalents in the south and north and the equilibrium heterozygosity for $u=10^{-4}$ and $10^{-5}$, given $S=0.083, h=0.02, s=1$ and $x_{2}=0.6$. For $u=10^{-4}, H=0.00392$ and the estimate of $n^{*}$ is 2181 and 1438 in the south and north, respectively, somewhat lower than the number of 5000 often given for Drosophila. On the other hand, for $u=10^{-5}, H=0.000402$ and the estimate of $n^{*}$ is 22244 and 14005 in the south and north, respectively, higher than the Drosophila estimate. The simplest explanation is that the average mutation rate is somewhere between $u=10^{-4}$ and $10^{-5}$ (this is consistent with Lande et al., 1994 and Kärkkäinen et al., 1996) and that the number of loci capable of producing recessive lethal mutations is intermediate between these estimates. We should note that some previous estimates have been much lower (Koski, 1971) or much higher (Namkoong \& Bishir, 1987).

\section{Possible explanations for the difference in lethal equivalents in the south and north}

As a baseline, let us determine the equilibrium heterozygosity for a given set of parameters that are reasonable for the southern samples, and then examine how changes in these values may influence the equilibrium heterozygosity (the relative patterns are similar for other levels of these parameters). Let us assume that the fitness of a homozygote is zero, i.e. $s=1$, the level of dominance is $h=0.02$ and the mutation rate is $10^{-4}$. Assume that the estimated level of selfing in the south is
0.083 (Kärkkäinen et al., 1996) and the estimated level of polyembryony is described by $x_{2}=0.6$. With these assumptions, $H_{\mathrm{e}}=0.003916$ in the south.

Let us now determine how large a change is necessary from these values to result in a $34.1 \%$ reduction in equilibrium heterozygosity, as found for the difference in lethals equivalents between the northern and southern samples (Table 3). First, using the expressions above, small changes in two factors can result in this reduction, i.e. an increase in dominance from $h=0.02$ to $h=0.054$ and an increase in selfing from $S=0.083$ to $S=0.146$ (note that the estimate of the primary selfing rate in the north is 0.192 , larger than this value), making these likely factors to be involved. Further, as we will discuss below there is related evidence supporting these factors.

Secondly, two other factors - a reduction in the mutation rate and a selfing pulse - can also result in this change. From iteration of the equations above, a reduction in the mutation rate of $34 \%$ will result in a similar reduction in the equilibrium heterozygosity. Also a selfing pulse of $S=0.30$ for five generations will result in a similar reduction in heterozygosity. However, as soon as the selfing pulse is discontinued, the heterozygosity level quickly begins to increase (see below).

Thirdly, three other factors - a bottleneck, finite population size, and stronger selection in the north against homozygotes - can also result in a heterozygosity reduction, but appear to be unlikely explanations. To determine the effect of a bottleneck and finite population size, the average of 5000 Monte Carlo simulations to generate genetic drift and the above expressions were utilized. From these simulations, a fivegeneration bottleneck of three individuals or a constant population size of 125 were necessary to result in the appropriate reduction in heterozygosity. Both of these conditions appear rather stringent (see discussion below). To determine the effect of homozygous selection, it was assumed that $s=1$ in the north and then the level of selection in the south necessary to result in a difference of $34.1 \%$ in heterozygosity was determined. In this case, it is necessary to have $s=0.58$ in the south for the needed difference. Finally, a decrease in the number of embryos to only one per ovule $\left(x_{1}=1\right)$, the maximum change possible, was insufficient to reduce the heterozygosity level by $34.1 \%$.

It is also important to examine how quickly these factors can result in a change of lethal equivalents, remembering that Scots pine reached the northernmost areas of Finland only 8000 years ago (Hyvärinen, 1976). Using the life expectancy data of Sarvas (1962), this was $\approx 100$ generations ago (Kärkkäinen et al., 1996). Again using the expressions above, as well as simulations for the bottleneck and finite population size, the pattern of 
Table 3 The possible causes and their evaluation for the difference in lethal equivalents in populations of Scots pine population from the south and north of Finland

\begin{tabular}{lccc}
\hline & \multicolumn{2}{c}{ Necessary change } & \\
\cline { 2 - 3 } Cause & South & North & Likelihood \\
\hline (a) Heterozygous selection & $h=0.02$ & $h=0.054$ & High \\
(b) Selfing & $S=0.083$ & $S=0.146$ & High \\
(c) Mutation rate & $10^{-4}$ & $0.66 \times 10^{-4}$ & Medium \\
(d) Selfing pulse & $S=0.083$ & Five gen. with $S=0.30$ & Medium \\
(e) Bottleneck & $N_{\mathrm{e}}=\infty$ & Five gen. with $N_{\mathrm{e}}=3$ & Low \\
(f) Population size & $N_{\mathrm{e}}=\infty$ & $N_{\mathrm{e}}=125$ & Low \\
(g) Homozygous selection & $S=0.58$ & $S=1$ & Low \\
(h) Polyembryony & $x_{1}=0.4$ & Not possible with $x_{1}=1$ & None \\
\hline & & &
\end{tabular}

heterozygosity was monitored over time. Figure 4 gives the expected time course of heterozygosity as a result of differences in selection against heterozygotes (selfing and mutation as given in Table 3) and shows that it is very possible for these factors to influence heterozygosity in 100 generations or fewer. Further, a difference in selection against homozygotes (not shown) would result in a pattern of heterozygosity very similar but slightly slower than that for mutation, so it could also result in the heterozygosity pattern in this time period.

Figure 5 demonstrates the expected change over 100 generations for a selfing pulse, a bottleneck, and population size for the values given in Table 3. For both the bottleneck and selfing pulse, the population would have recovered to the initial levels by 100

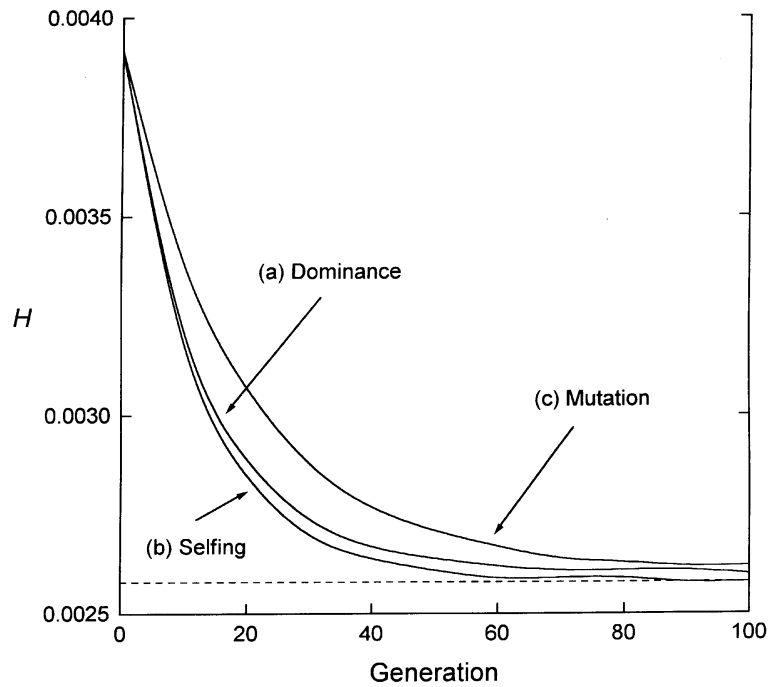

Fig. 4 The heterozygosity over 100 generations based on the equilibrium in the south with $h=0.02, S=0.083, u=10^{-4}$, $s=1$ and $x_{2}=0.6$ in an infinite population resulting from changes in (a) selection against heterozygotes (dominance), (b) selfing and (c) mutation. generations or fewer. The recovery from the selfing pulse occurs immediately after the pulse ceases and is initially similar to the decline and then asymptotes to the original equilibrium. On the other hand, the greatest average effect of a bottleneck does not occur immediately after the bottleneck, but is delayed to between generations $10-15$ and then only recovers at a rate slower than the decline. Because of the low frequency of the lethal, the bottleneck generally results in loss of the lethal but does result in an increase (mostly to 0.167 with three individuals) in some replicates. The higher frequencies are rapidly reduced by selection, whereas the restoration of the lethal in the majority of replicates by mutation occurs more slowly, so that the average declines for a number of generations before increasing

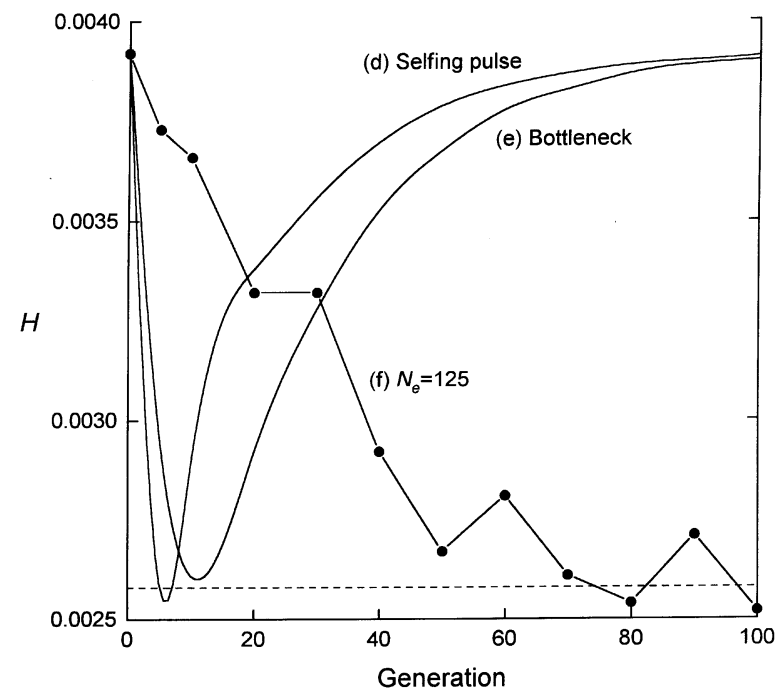

Fig. 5 The change in heterozygosity based on the equilibrium in the south with $h=0.02, S=0.083, u=10^{-4}, s=1$ and $x_{2}=0.6$ in an infinite population resulting from changes in (d) a selfing pulse, (e) a bottleneck and (f) finite population size.

(C) The Genetical Society of Great Britain, Heredity, 82, 441-450. 
back to the equilibrium. The decline in heterozygosity for the finite population size to the lower value occurs over about 50 generations and then varies considerably, even though the results given here are the average of 5000 replicates. Recovery, given a return to infinite population size after the finite size, is like that for the bottleneck example and would occur fairly quickly.

How robust are these effects in the face of gene flow from the south? To obtain a general idea of the impact of gene flow, let us assume that $s=1.0$ (lethality) and $x_{1}=1.0$ (no polyembryony). The changes in genotypic frequencies are then

$$
\begin{aligned}
& P^{\prime}=\frac{(1 \quad u)}{\bar{w}}\{S(1 \quad u)(P+H / 4) \\
& \left.+\left(\begin{array}{ll}
1 & S
\end{array}\right) p\left[p(1 \quad u)(1 \quad m)+p_{m} m\right]\right\}
\end{aligned}
$$

and

$$
\begin{aligned}
& H^{\prime}=\frac{(1 \quad h)}{\bar{w}}\{S(1 \quad u)[2 u P+(1+u) H / 2] \\
& +\left(\begin{array}{ll}
1 & S
\end{array}\right)\left\{p(1 \quad u)\left[(q+u p)(1 \quad m)+q_{m} m\right]\right. \\
& \left.\left.\left.+\left[\begin{array}{ll}
1 & u
\end{array}\right)(1 \quad m)+p_{m} m\right](u p+H / 2)\right\}\right\},
\end{aligned}
$$

where $m$ is the level of pollen gene flow from the south, $p_{\mathrm{m}}$ and $q_{\mathrm{m}}$ are the allelic frequencies of $A_{1}$ and $A_{2}$ in the migrant pollen from the south, and $\bar{w}$ is the sum of the right side of the equations when multiplied by $\bar{w}$.

Let us assume that the south is at equilibrium for $S=0.083, h=0.02$, and $u=10^{-4}, \quad$ so that $q_{\mathrm{m}}=0.00157$. If the level of gene flow from the south is low, say $m=0.01$ or less, then there is little impact on the frequency in the north, i.e. there is still a 32 to $33 \%$ difference when there is a change in dominance, selfing or mutation, almost as large as the $34 \%$ without gene flow. When the gene flow is higher, say $m=0.1$, the impact is higher, but there is still approximately a $25 \%$ reduction from the south. It seems unlikely that the gene flow from the south would be this large and that most of the gene flow would be from some intermediate populations in which the allele frequency may also be intermediate between south and north. Overall, it appears that it would be quite possible to retain the observed difference in inbreeding depression in the face of gene flow.

\section{Discussion}

In recent years, evidence of variation in the extent of inbreeding depression in related species and even in different populations of the same species has been accumulating. A number of evolutionary factors could potentially be involved in any such differences but it is difficult to understand or predict their impacts without a detailed analytical or numerical examination. Here we have attempted to explain the higher number of lethal equivalents in southern Finland populations of Scots pine than in northern Finland populations, by determining the impact of differences in eight different factors that could influence the frequency of lethal or detrimental alleles. Similar systematic approaches should prove useful in other examples where there are genetic load differences among related species or populations of the same species. For example, some other species of pines appear to have a low number of embryonic lethals (e.g. Williams \& Savolainen, 1996).

Before we discuss the apparent impact of the various factors in explaining the differences in the estimated number of lethal equivalents in the south and north Finland populations of Scots pine, let us summarize several other findings. First, the impact of polyembryony (two embryos per ovule) compared to one embryo is to increase the expected equilibrium frequency of lethal and detrimental alleles (as well as heterozygosity and number of lethal equivalents) by $\approx 41 \%$ when there is no selfing and $\approx 33.3 \%$ when the rate of selfing is greater than 0.05 . This occurs because selection is somewhat relaxed against heterozygotes because polyembryony gives an additional opportunity to produce a viable embryo. Secondly, the expected level of heterozygosity for a recessive lethal when the selfing is greater than 0.05 with one embryo is $\approx 4 u / S$, and with two embryos is $\approx 16 u / 3 S$. Therefore, an increase in selfing or a decrease in mutation rate (above a certain level of selfing) results in a linear decrease in equilibrium heterozygosity. On the other hand, an increase in the level of selfing from $S=0$ to $S=0.05$ results in a large reduction in heterozygosity for all levels of polyembryony. Finally, an increase in the level of dominance from complete recessivity ( $h=0)$ to some selection against the heterozygote (say $h=0.02$ ) results in a substantial reduction in heterozygosity.

What is the most likely cause of the reduced genetic load in the northern populations? To preface the following discussion, it is entirely possible that several factors may combine to result in the reduction. Two factors, an increase in self-fertilization and increased selection against heterozygotes, stand out as explanations based on the theoretical examination here and the biological evidence (Kärkkäinen et al., 1996).

The density of trees in the northern forests is less than that in the southern ones. In addition, pollen production in the north is less even and the quantities are lower than in the southern stands. Therefore, the average pollen production per square metre is three times lower in the northern forests than in the southern populations (Koski $\&$ Tallqvist, 1978). In wind-pollinated plants, the rate of 
self-fertilization is assumed to be higher when the concentration of self pollen is higher around the individual (mass-action assumption, see Ziehe \& Gregorius, 1988; Holsinger, 1991). Lower pollen production in the north can thus be a reasonable explanation for an increase in observed rate of primary self-fertilization in the north (from 0.083 to 0.192 ) and this difference, based on our theoretical examination, is more than enough to result in the difference in the genetic load in the two populations.

The northern populations grow in much more harsh environmental conditions where, for example, the growing season is significantly shorter than in the south (mean annual temperature sum is 1100-1300 degrees in the south and less than 900 degrees in the north, Harju et al., 1996). Because of the short growing season in the north, seed maturation in particular is a great problem in most years, whereas the growing season in the south is long enough for complete seed maturation nearly every year. If embryonic lethals even slightly reduced the rate of seed development as heterozygotes, then this would be of little selective significance in the south but would be of great selective importance in the north, effectively resulting in complete recessivity in the south and $h>0$ in the north. As shown theoretically above, a small increase in dominance from $h=0.02$ in the south to $h=0.054$ in the north would be enough to explain the difference in inbreeding depression.

Although other factors may explain the differences between the south and north, they all seem less likely. For example, there have been no indications that the mutation rate would be lower in the north. Given that the north is a very stressful environment, it could be suggested that the mutation rate would be higher there. Or if the mutation rate is a function of the number of cell divisions per generation, fewer cell divisions per generation in the north could be the cause, but there is also no evidence of this. Although a selfing pulse of five generations with $S=0.30$ does not seem very extreme if the estimated primary selfing rate in the north is 0.192 , the effect of such a pulse is ephemeral and would have had to have occurred in the past 10 generations or so for its effects to be still present. Scots pine forms large, nearly continuous populations over much of Finland and the very high gene flow from pollen results in a large effective population size with little differentiation from south to north for neutral markers (Muona, 1990; Karhu et al., 1996). On the other hand, strong selective differences can result in differentiation in spite of this extensive gene flow (Karhu et al., 1996). In other words, it seems quite unlikely that the effective population size is as little as 125 when there are several hundred or more adult Scots pines per hectare over millions of hectares in Finland. Similarly, it seems quite unlikely that there could have been a recent bottleneck of three individuals for five generations in the recent past. Even if this did happen, the gene flow should erase such a random event fairly quickly (gene flow should have a similar effect on a selfing pulse). Finally, much stronger selection against homozygotes could also result in the observed reduction. However, the proportional change is more than 12 times as large as the change necessary in the fitness of the heterozygotes to have the same effect. The effect of biparental inbreeding has not been examined here because there is little family structure in pines (Epperson \& Allard, 1989; Latta \& Mitton, 1997).

\section{Acknowledgements}

We appreciate the support of Research Council for Agriculture and Forestry and the Environment and Natural Resources Council of Finland, and thank Russ Lande for comments on the manuscript.

\section{References}

CHARLESWORTH, D. AND CHARLESWORTH, B. 1987. Inbreeding depression and its evolutionary consequences. Ann. Rev. Ecol. Syst., 18, 237-268.

EPPERSON, B. K. AND ALLARD, R. W. 1989. Spatial autocorrelation analysis of the distribution of genotypes within populations of lodgepole pine. Genetics, 121, 369-377.

GRIFFIN, A. R. AND LINDGREN, D. 1985. Effect of inbreeding on production of filled seed in Pinus radiata - experimental results and a model of gene action. Theor. Appl. Genet., 71, 334-343.

HARJU, A. M., KÄRKKÄINEN, K. AND RUOTSALAINEN, S. 1996. Phenotypic and genetic variation in the seed maturity of Scots pine. Silvae Genet., 45, 205-211.

HEDRICK, P. W. 1985. Genetics of Populations. Jones and Bartlett, Boston, MA.

HOLSINGER, K. E. 1991. Mass-action models of plant mating systems: the evolutionary stability of mixed mating systems. Am. Nat., 138, 606-622.

HUSBAND, B. C. AND SCHEMSKE, D. W. 1996. Evolution of the magnitude and timing of inbreeding depression in plants. Evolution, 50, 54-70.

HYVÄRINEN, H. 1976. Flandrian pollen deposition rates and tree line history in northern Fennoscandia. Boreas, 5, 163-175.

KARHU, A., HURME, P., KARJALAINEN, M., KARVONEN, P., KÄRKKÄINEN, K., NEALE, D. AND SAVOLAINEN, O. 1996. Do molecular markers reflect patterns of differentiation in adaptive traits of conifers? Theor. Appl. Genet., 93, 215-221.

KÄRKKÄINEN, K., KOSKI, V. AND SAVOLAINEN, O. 1996. Geographical variation in the inbreeding depression of Scots pine. Evolution, 50, 111-119.

Koski, v. 1971. Embryonic lethals of Picea abies and Pinus sylvestris. Comm. Inst. For. Fenn., 75, 1-30.

KOSKI, V. AND TALLQVIST, R. 1978. Results of long-time measurements of the quantity of flowering and seed crop of forest trees. Folia Forestalia, 363, 1-60 (in Finnish with English summary). 
LANDE, R. AND SCHEMSKE, D. W. 1985. The evolution of selffertilization and inbreeding depression in plants. I. Genetic models. Evolution, 39, 24-40.

LANDE, R., SCHEMSKe, D. W. AND SCHUlTz, S. T. 1994. High inbreeding depression, selective interference among loci, and the threshold selfing rate for purging recessive lethal mutations. Evolution, 48, 965-978.

LATTA, R. G. 1995. The effects of embryo competition with mixed mating on the genetic load in plants. Heredity, 75, 637-643.

LATTA, R. G. AND MITTON, J. B. 1997. A comparison of population differentiation across four classes of gene marker in limber pine (Pinus flexilis James). Genetics, 146, 1153-1163.

MUONA, O. 1990. Population genetics in tree improvement. In: Brown, A. H. D., Clegg, M. T., Kahler, A. L. and Weir, B. S. (eds) Plant Population Genetics, Breeding, and Genetic Resources, pp. 282-298. Sinauer Associates, Sunderland, MA.

NAMKOONG, G. AND BISHIR, J. 1987. The frequency of lethal alleles in forest tree populations. Evolution, 41, 1123-1127.

ORR-EWING, A. L. 1957. A cytological study of the effects of selfpollination in Pseudotsuga menziesii. Silvae Genet., 6, 179185.
PARK, Y. S. AND FOWLER, D. P. 1982. Effects of inbreeding and genetic variances in a natural population of Tamarack (Larix decidua (Du Roi) K. Koch) in eastern Canada. Silvae Genet., 31, 21-26.

SARVAS, R. 1962. Investigations on the flowering and seed crop of Pinus sylvestris. Comm. Inst. For. Fenn., 53, 1-198.

SAVOLAINEN, O., KÄRKKÄINEN, K. and KUITTINEN, H. 1992. Estimating numbers of embryonic lethals in conifers. Heredity, 69, 308-314.

SIMMONS, M. J. AND CROW, J. F. 1977. Mutations affecting fitness in Drosophila populations. Ann. Rev. Genet., 11, 49-78.

SORENSEN, F. C. 1969. Embryonic genetic load in coastal Douglas fir, Pseudotsuga menziesii var. menziesii. Am. Nat., 103, 389-398.

WILLIAMS, C. G. AND SAVOLAINEN, O. 1996. Inbreeding depression in conifers: implications for breeding strategy. For. Sci., 42, 102-117.

ZIEHE, M. AND GREGORIUS, H.-R. 1988. Selection caused by selffertilization. II. Ecological constraints on selfing advantage. J. Evol. Biol., 1, 233-253. 